\title{
Design of a New Switching Power Supply for the ATLAS TileCal Front-End Electronics
}

\section{TWEPP 2012}

Gary Drake

Senior Engineer

Argonne National Laboratory, USA

On Behalf of the ATLAS Tile Calorimeter System

Oxford, England

Sept. 20, 2012 


\section{TileCal in the ATLAS Detector}
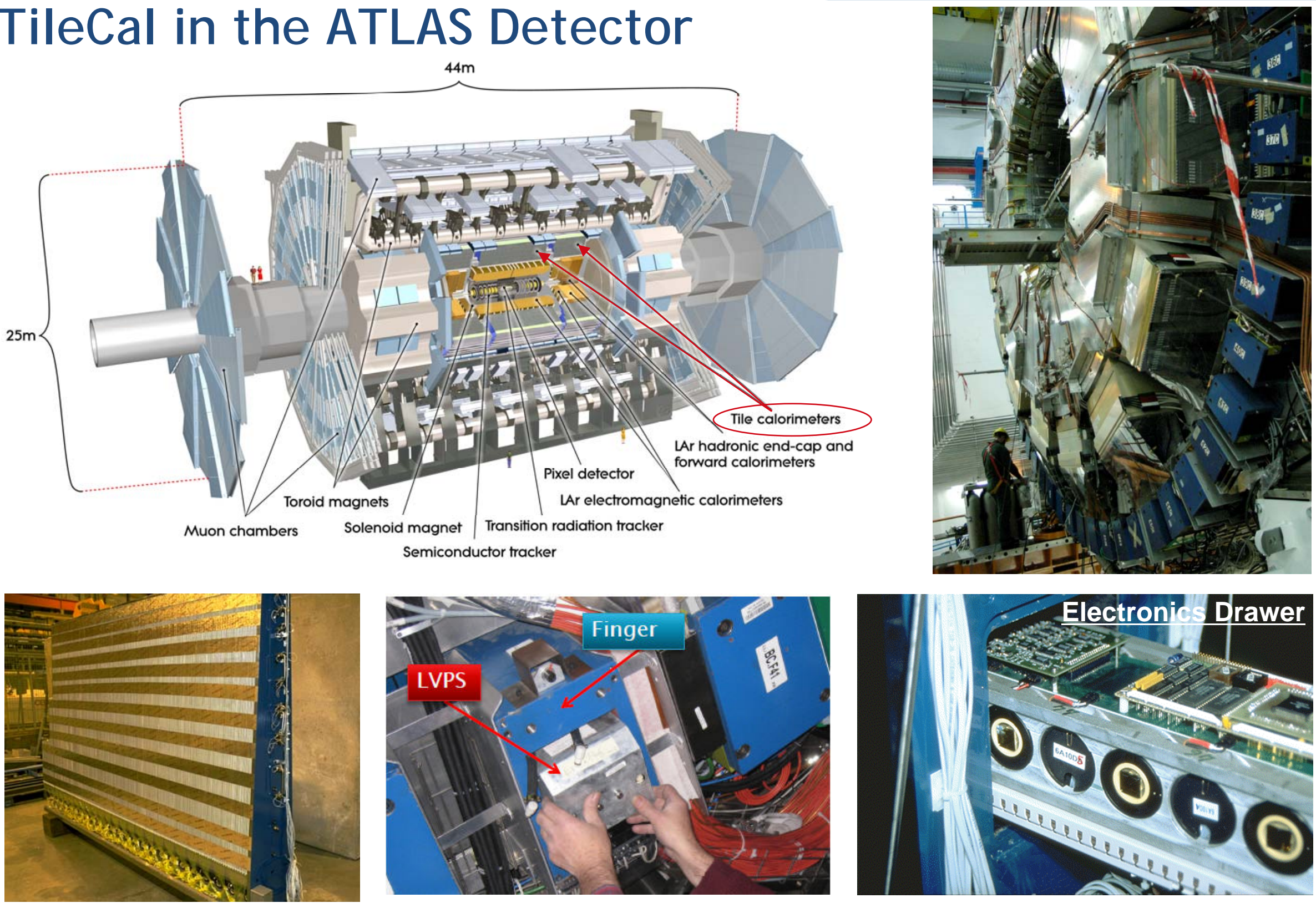

$\Rightarrow$ There are 256 modules in the detector, $\sim 10,000$ readout channels Switching Power Supply for ATLAS TileCal - G. Drake - TWEPP - Oxford, England - Sept. 20, 2012 


\section{Block Diagram of the TileCal Power Dist. System}

- Block Diagram of the TileCAL Power Distribution System:

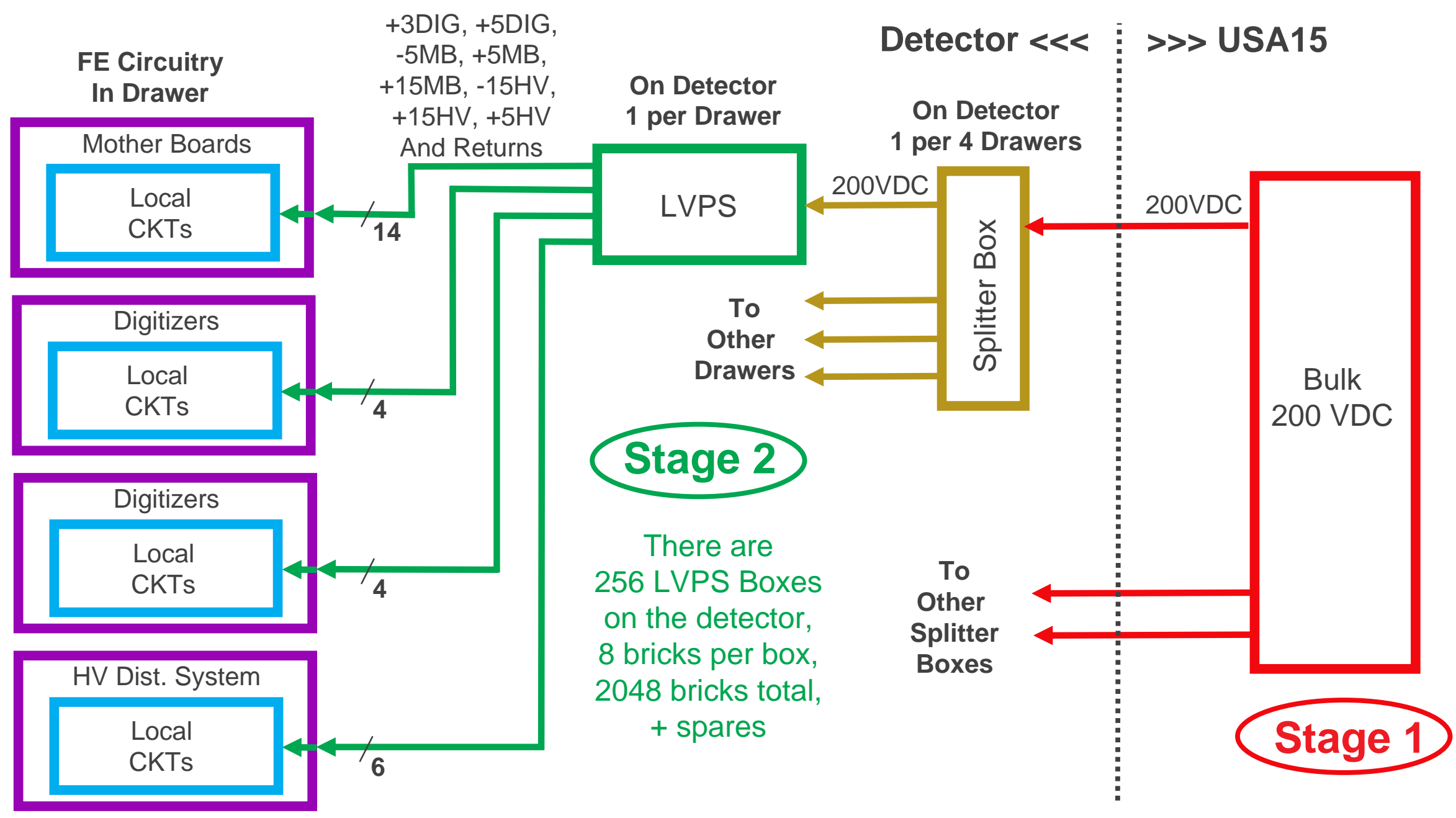

Switching Power Supply for ATLAS TileCal - G. Drake - TWEPP - Oxford, England - Sept. 20, 2012 


\section{The ATLAS TileCal Low Voltage Power Supply (LVPS)}

- Block Diagram of the LVPS Box:

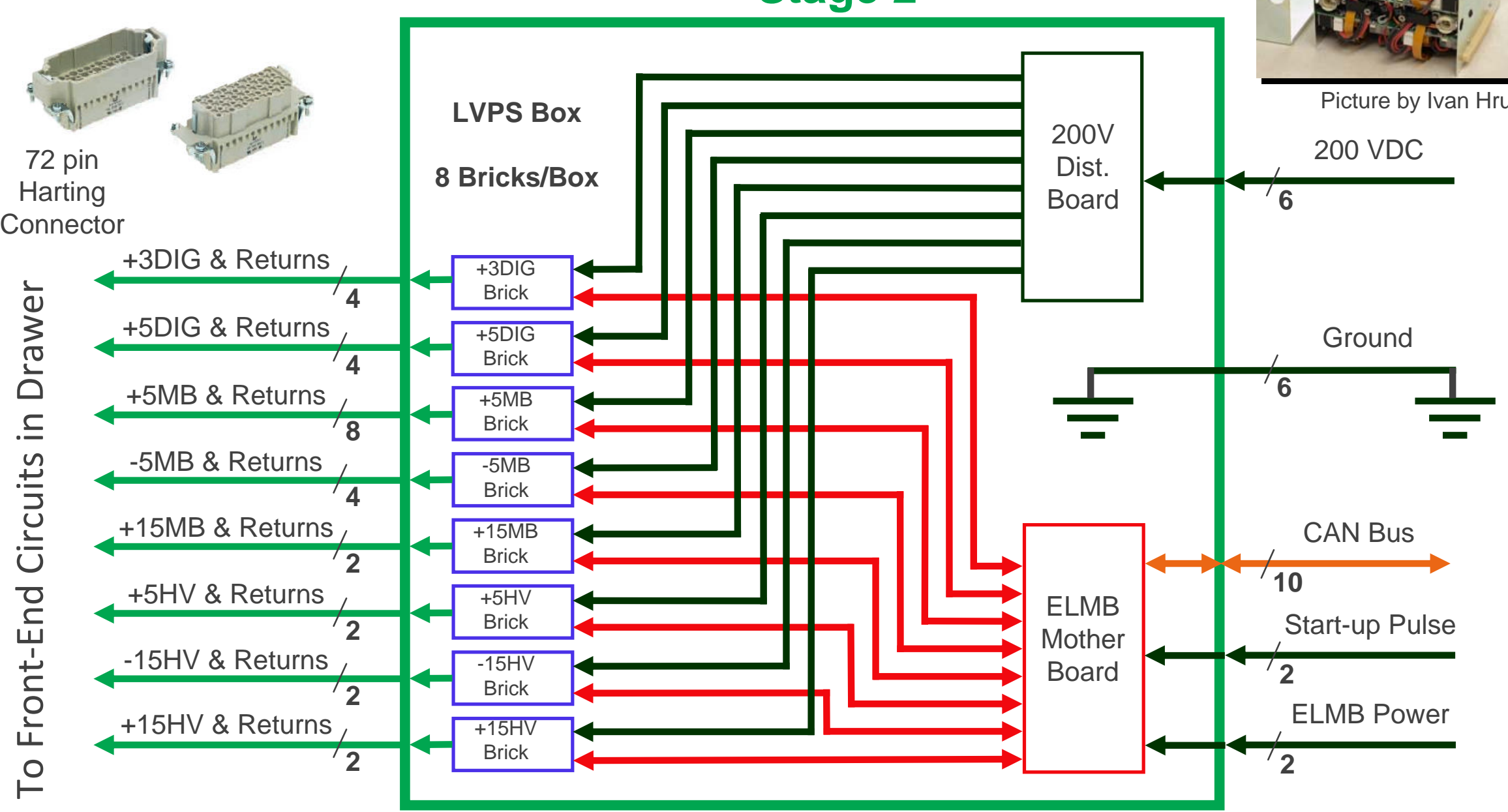

Range of Voltages: $\quad 5: 1$

Range of Currents: $62: 1$
Stage 2
$\Rightarrow$ One Basic Brick Design

Different Component Values

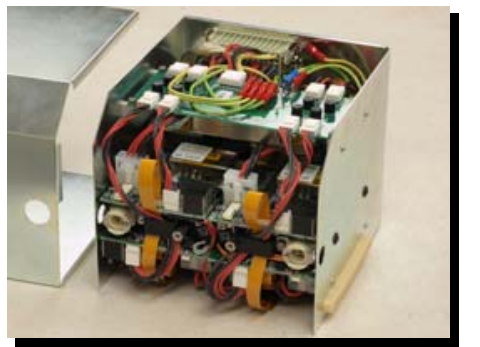

Picture by Ivan Hruska

200 VDC

6

$\Rightarrow$ Note: No Redundancy...

Switching Power Supply for ATLAS TileCal - G. Drake - TWEPP - Oxford, England - Sept. 20, 2012 


\section{The ATLAS TileCal LVPS (Cont. )}

- Full Custom Design

- Novel switching DC-DC power supply; $300 \mathrm{KHz}$

- Original design by Ivan Hruska

- Features:

- Custom, compact, high-efficiency, 250 Watt

- 8 different voltages $\rightarrow$ Customized bricks

- Water cooled; System interface \& monitoring

- Environment: Magnetic field; Radiation tolerant

- 256 boxes on detector, 2048 bricks, + spares

- Reliability is Important $\rightarrow$ Infrequent Access

\begin{tabular}{|c|r|r|}
\hline Brick Type & Nominal Voltage & Nominal Current \\
\hline$-5 \mathrm{MB}$ & -5.3 & $5.6 / 4.2$ \\
\hline$+5 \mathrm{MB}$ & +5.4 & $11.1 / 8.3$ \\
\hline$+15 \mathrm{MB}$ & +14.5 & $0.45 / 0.34$ \\
\hline$+3 \mathrm{DIG}$ & +3.45 & $4.74 / 3.56$ \\
\hline$+5 \mathrm{DIG}$ & +5.30 & $5.6 / 4.2$ \\
\hline -15HV & -14.5 & $1.55 / 1.16$ \\
\hline$+5 \mathrm{HV}$ & +5.0 & $0.18 / 0.14$ \\
\hline$+15 \mathrm{HV}$ & +14.5 & $0.29 / 0.22$ \\
\hline
\end{tabular}

View of Box, Cover Removed

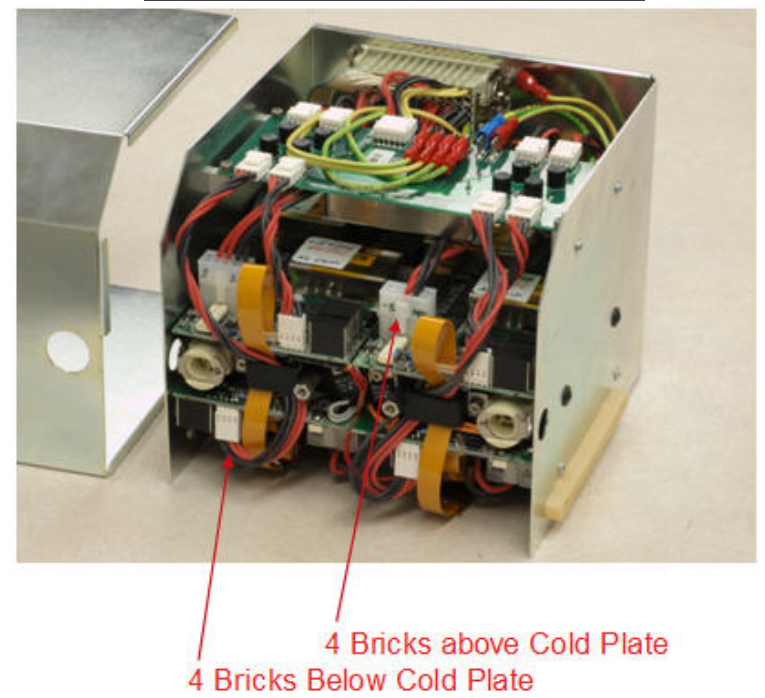

Side View of Box, Cover Removed

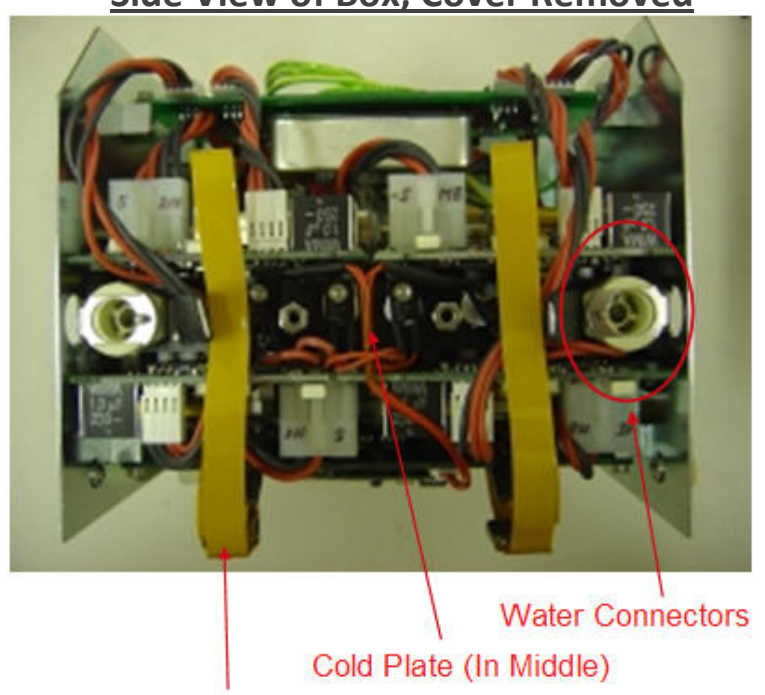

Flat Cable to ELMB (On Bottom)
Individual Brick (Not to Scale)

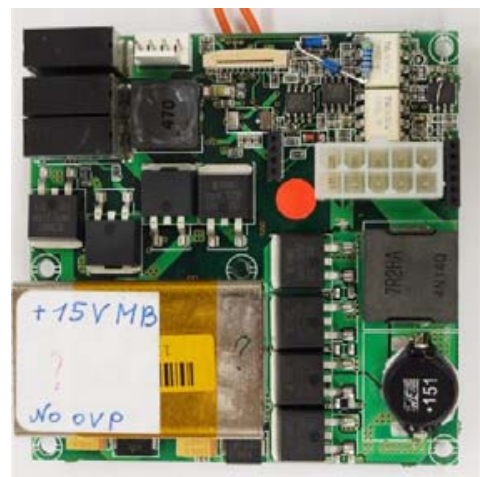

Pictures by B. Palan

Switching Power Supply for ATLAS TileCal - G. Drake - TWEPP - Oxford, England - Sept. 20, 2012 


\section{TileCal LVPS Redesign Project}

- Original production

- Produced in 2005

- Installed on detector in 2006-2007

- Performance \& reliability concerns developed:

- Board fabrication problems

\section{Post-assembly modifications}

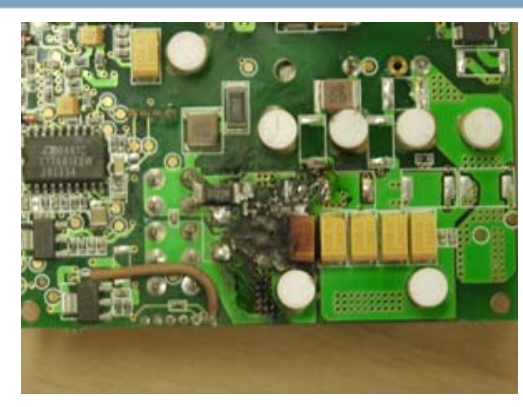

Burned capacitors

- Assembly \& soldering problems

- Substantial rework

- Stability problems

- Spontaneous tripping on detector $\rightarrow$ Noise

- Hard failures on detector - 6-10 per year

- Later - tripping correlated with beam

- Redesign project

- Community was concerned about long-term reliability

- Review recommendation in 2009: Consider new design

- ANL Elec. Eng. Group collaborating since 2006, took on redesign project

$\Rightarrow$ Thorough evaluation of problems \& testing

$\Rightarrow$ Conclusion: Redesign best way to address issues

$\Rightarrow$ Goals:

$\Rightarrow$ Address issues

$\Rightarrow$ Minimize system perturbation

$\Rightarrow$ Design "drop-in" replacement

Original Bricks: Spontaneous Tripping, Non-Destructive

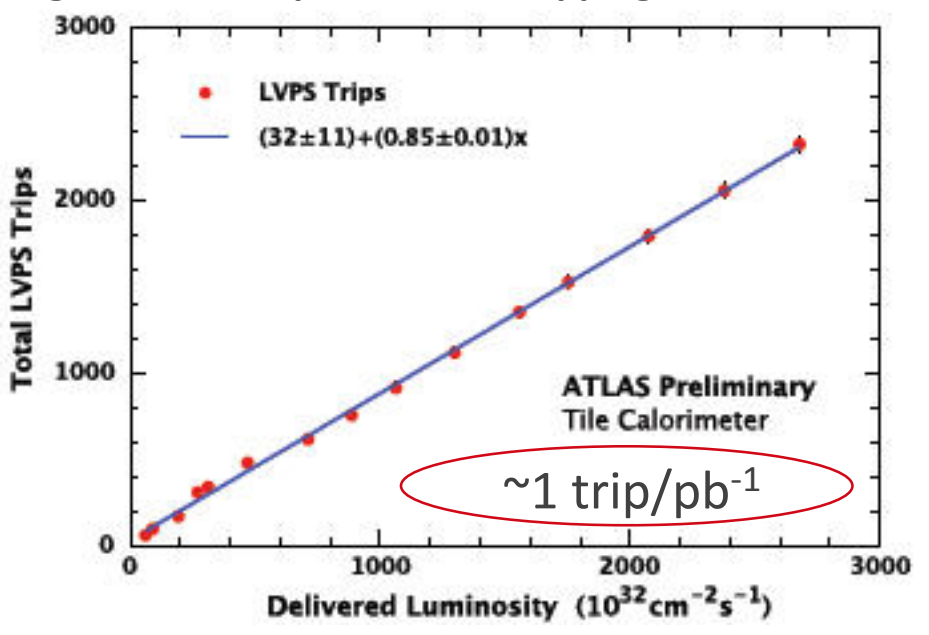

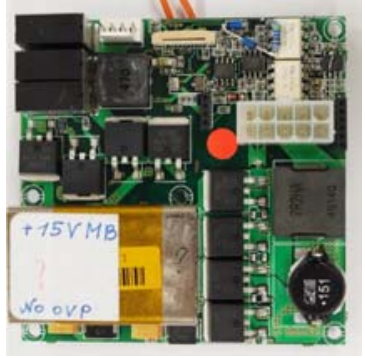

Original Brick

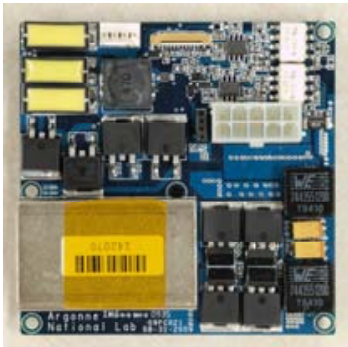

ANL“Blue Brick”

Switching Power Supply for ATLAS TileCal - G. Drake - TWEPP - Oxford, England - Sept. 20, 2012 
Brick Issues \& Improvements

Improved Reliability

Critical Issues

- Reduce Noise

- Opto-Isolator latch-up

- Thermal Management

- Better ESD protection of ICS

- Medium-Impact Issues

- Improve stability

- Improve trip circuitry

- Power sequencing

- Fabrication and soldering quality

- Capacitor Voltage Ratings

- Non-Critical But Highly Desirable

- Start-up pulse current

- Eliminate pre-loads

- Improve monitoring circuitry

- IPC specs for assembly

Improved

Performance
- Reduce/improve tuning

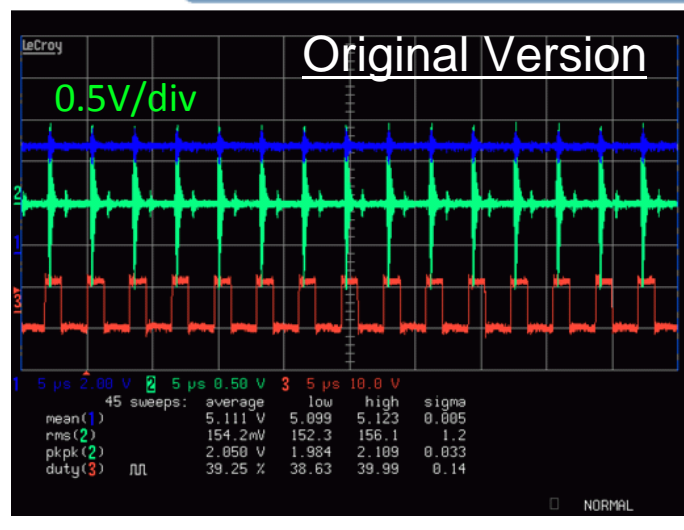

Output Noise

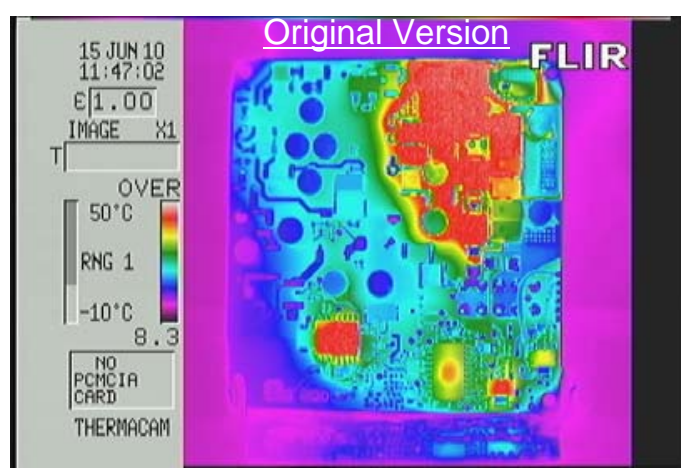

Thermal Image

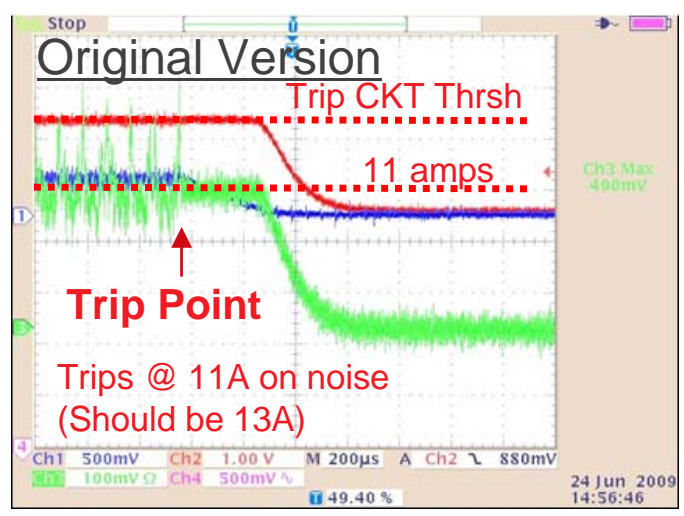

Tripping on

Noise

$\Rightarrow$ Improved noise performance will reduce tripping

$\Rightarrow$ Other improvements in reliability as well

Switching Power Supply for ATLAS TileCal - G. Drake - TWEPP - Oxford, England - Sept. 20, 2012 


\section{The ATLAS Tilecal LVPS (Cont. )}

- Block Diagram of New Brick

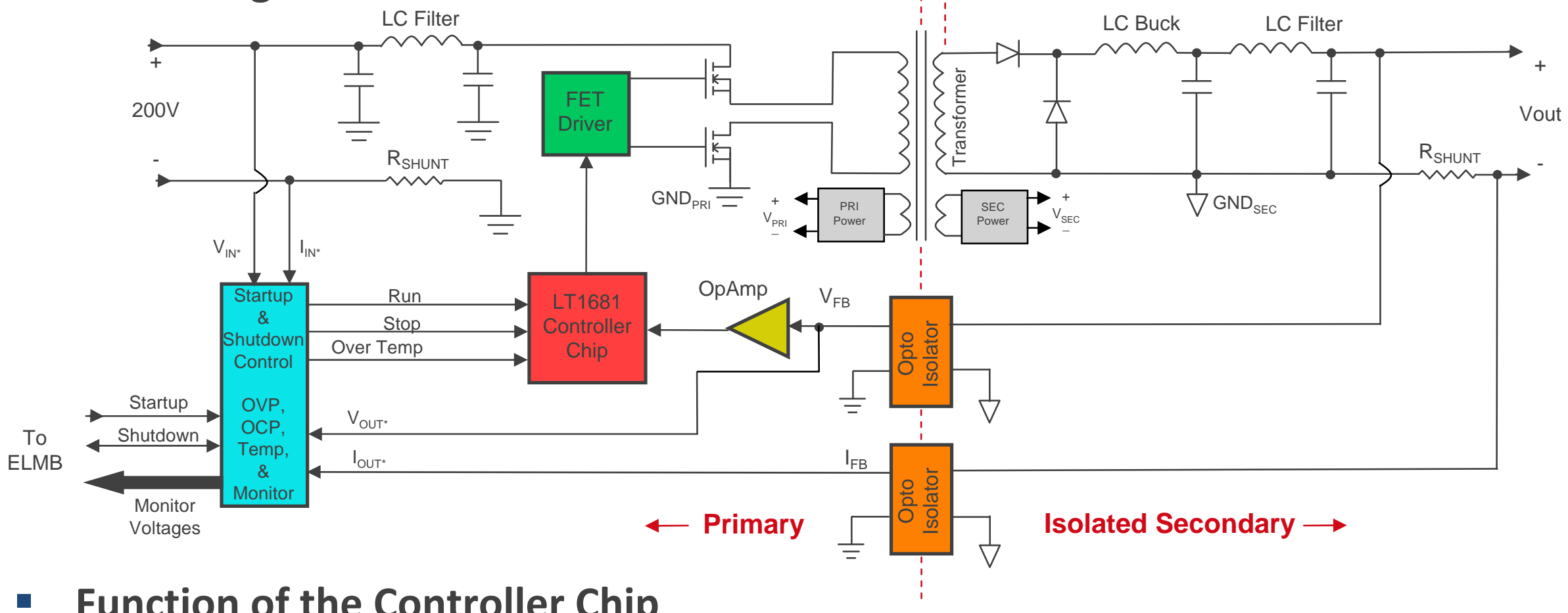

- Function of the Controller Chip

- Modulate the pulse width of the clock driving the switching, based on feedback

- When the output voltage is too high, reduce the pulse width

- When the output voltage is too low, increase the pulse width

Output Voltage Too Large
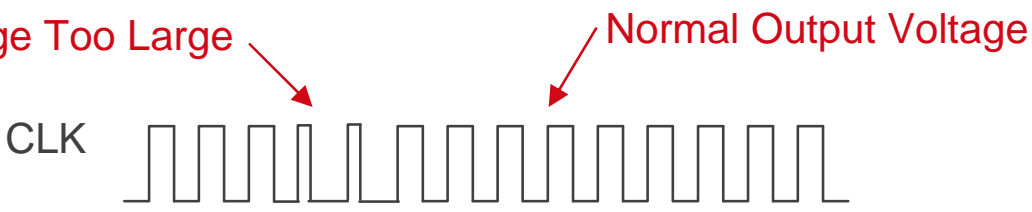

Switching Power Supply for ATLAS TileCal - G. Drake - TWEPP - Oxford, England - Sept. 20, 2012 


\section{Discussion of Improvements to Design}

- Critical Issue: Reduce Noise

$\Rightarrow$ What we did:

- Add/improve ground planes

- Add/improve filtering

- Careful attention to return currents
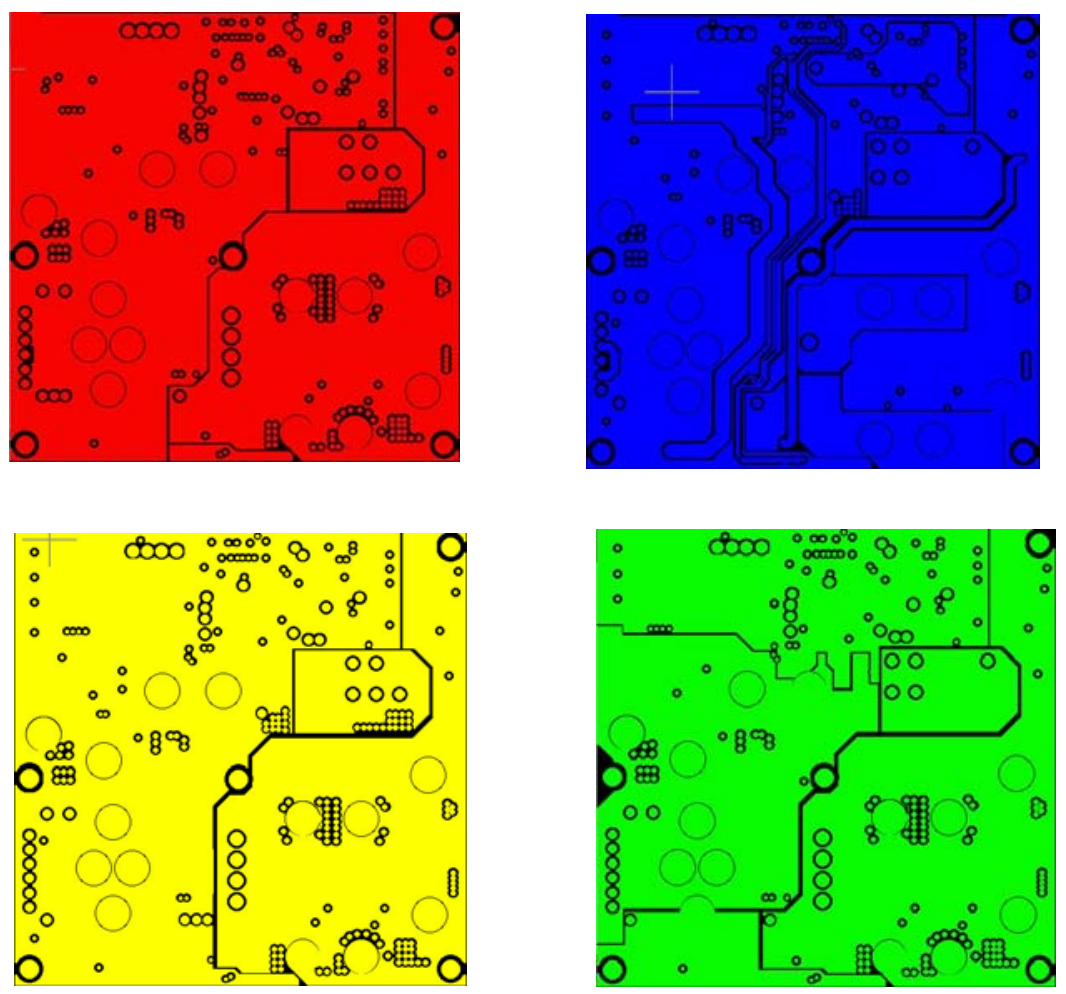

$$
\frac{\text { V7.3.0 - Internal Layers }}{\text { (Colors }=\mathrm{Cu})}
$$
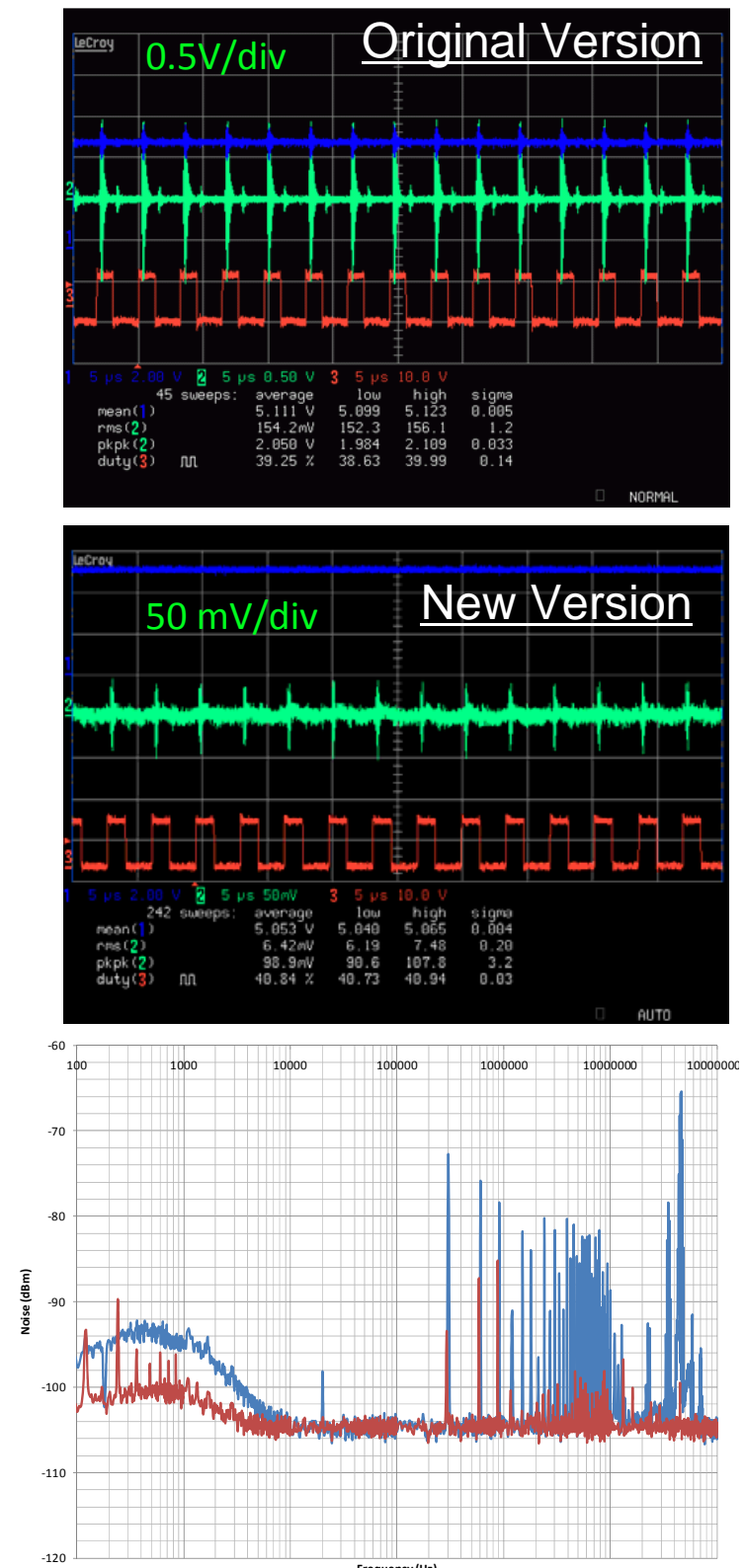

Output Noise •+5MB@13A

Old Version

New Version

Switching Power Supply for ATLAS TileCal - G. Drake - TWEPP - Oxford, England - Sept. 20, 2012 


\section{Improvements to Design (Cont.)}

- Critical Issue: Opto-Isolator Latch-up

$\Rightarrow$ The symptoms:

- Bricks would latch up spontaneously

- Can persist for a long time...
$\Rightarrow$ The cause:

- Undocumented requirement for extra bypass capacitors $\Rightarrow$ What we did:

- Add bypass capacitors to inputs

- Add bypass capacitors to power pins

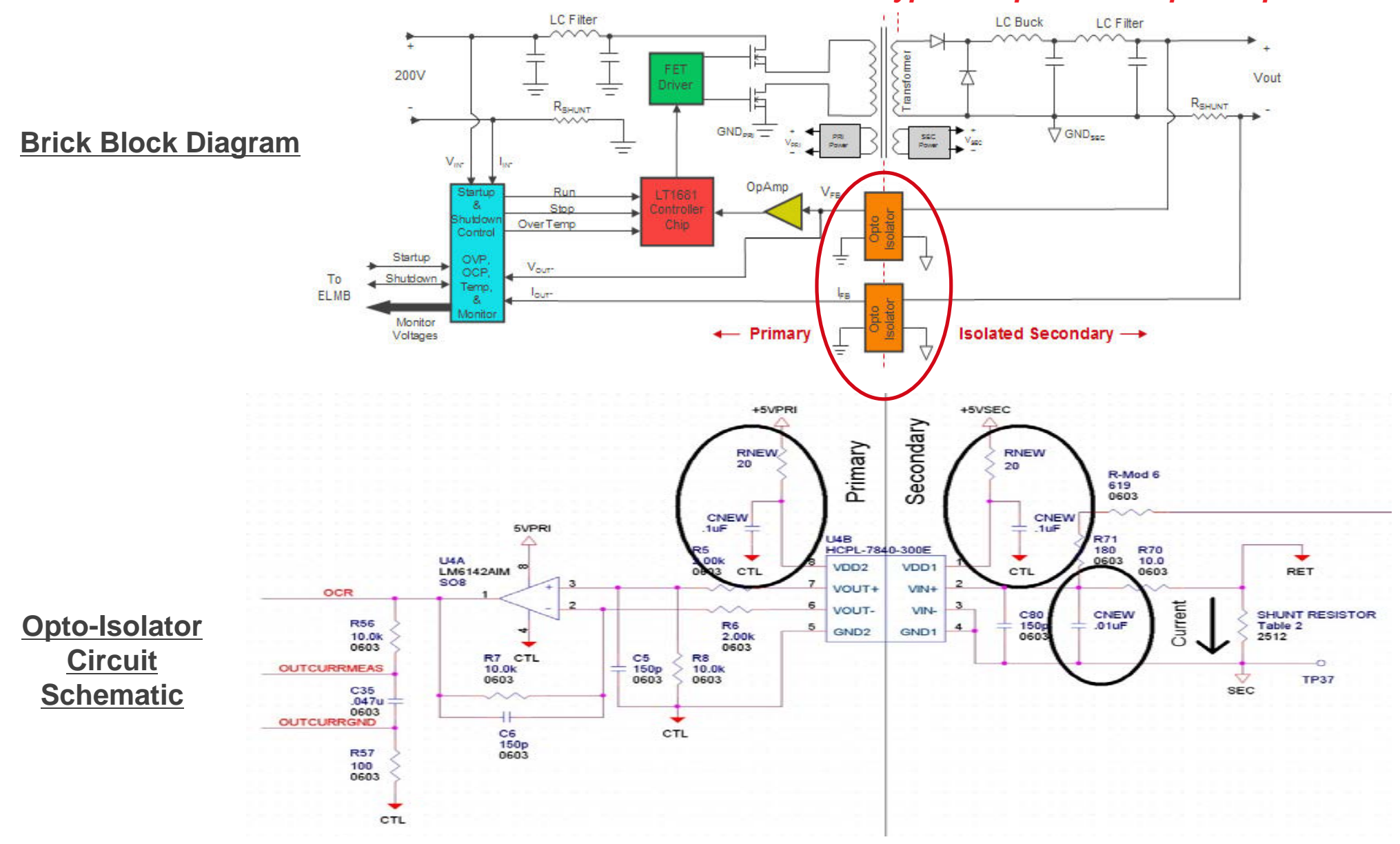

Switching Power Supply for ATLAS TileCal - G. Drake - TWEPP - Oxford, England - Sept. 20, 2012 


\section{Improvements to Design (Cont.)}

- Critical Issue: Thermal Management

$\Rightarrow$ The symptoms:

- U1 (controller chip) \& U2 (FET driver) had high failure rates
$\Rightarrow$ A possible cause:

- Insufficient thermal coupling to cold plate

$\Rightarrow$ What we did:

- New layout to get chips over cold plate

- Add thermal foam (Gap Pad) to couple chips to cold plate

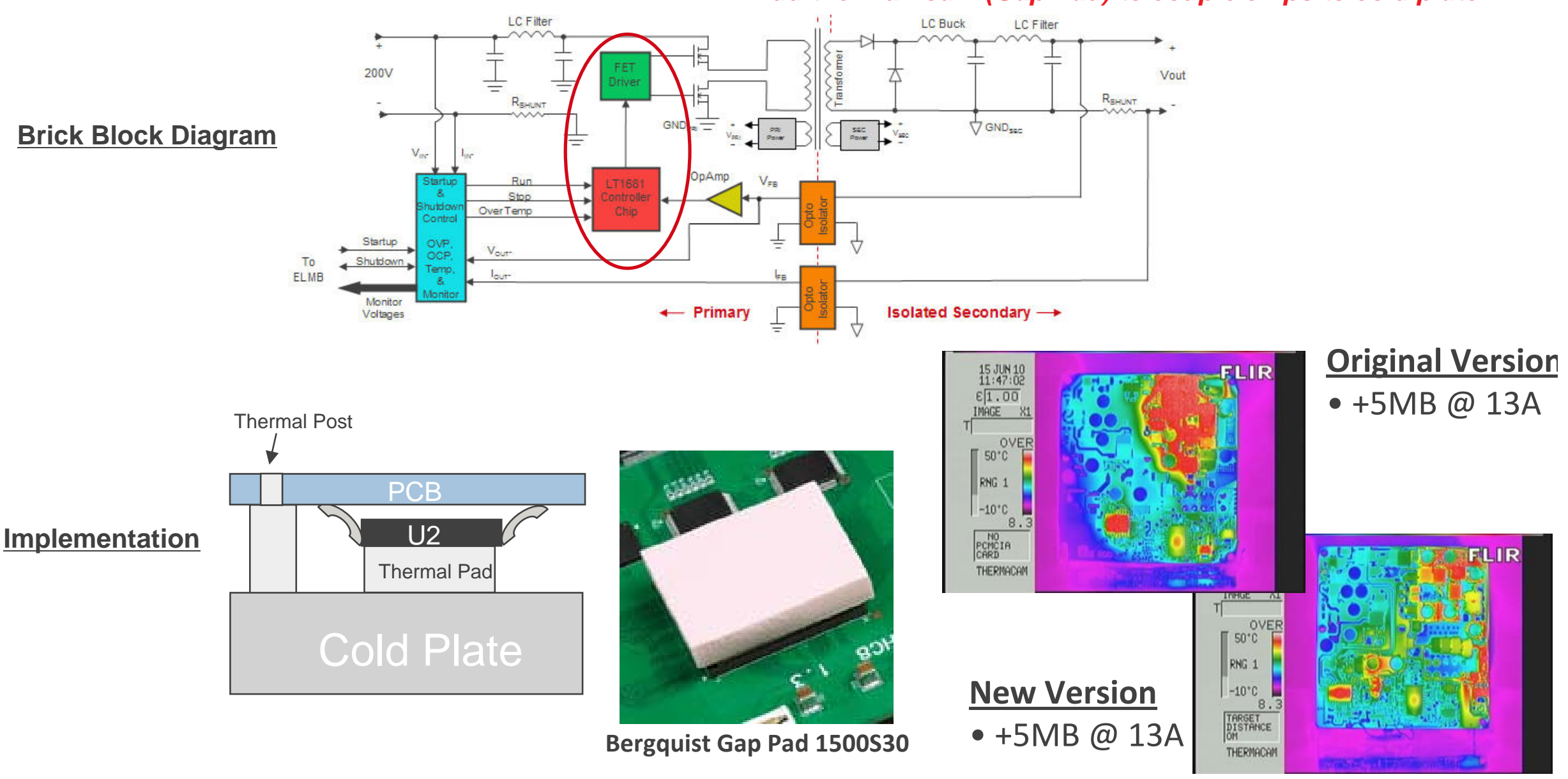

Switching Power Supply for ATLAS TileCal - G. Drake - TWEPP - Oxford, England - Sept. 20, 2012 


\section{Improvements to Design (Cont.)}

- Critical Issue: Overload protection of U2 (FET driver)

$\Rightarrow$ The symptoms:

- U2 (FET driver) had high failure rates
$\Rightarrow$ A possible cause:

- Overload on input pins ( $\rightarrow$ Noise?)

$\Rightarrow$ What we did:

- Add diode protection to input pins

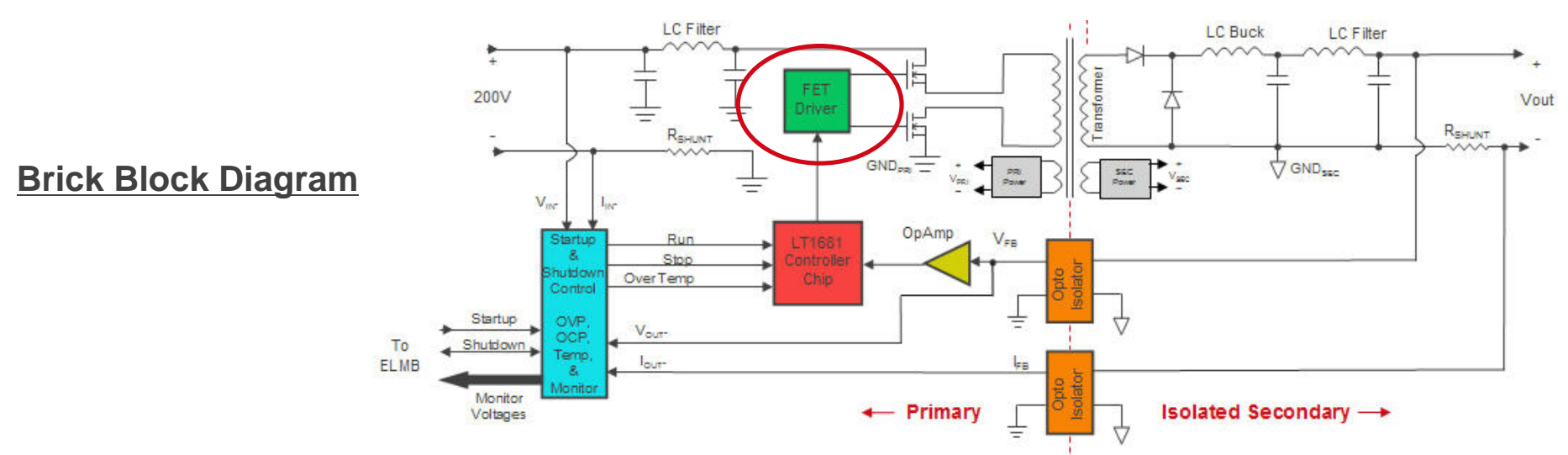

FET Driver

Circuit

Schematic

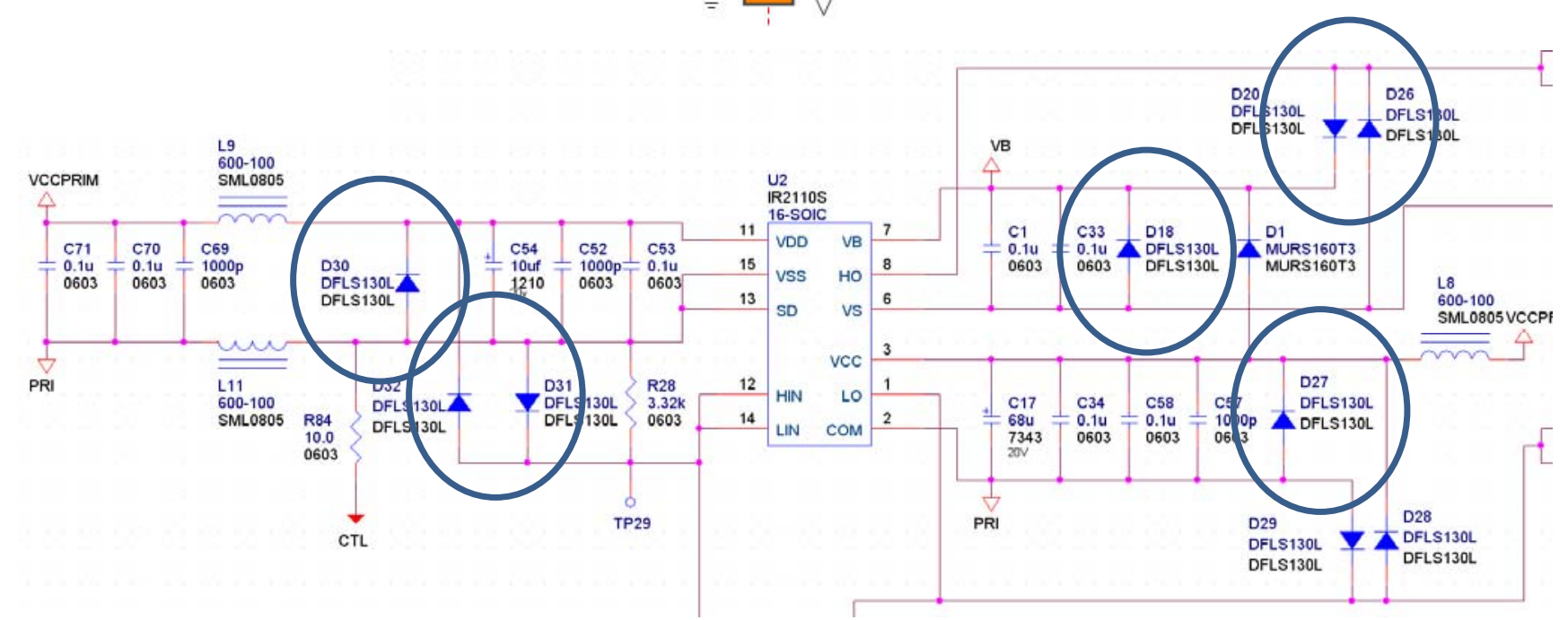

Switching Power Supply for ATLAS TileCal - G. Drake - TWEPP - Oxford, England - Sept. 20, 2012 


\section{Summary of Design Changes}

Improved

Reliability
$\uparrow$
Critical Issues
- What we have done:
- Reduce Noise.
$\Rightarrow$ More ground planes; improved filtering
- Opto-Isolators
$\Rightarrow$ Filter supply $\&$ input pins
- Thermal Management
$\Rightarrow$ U1 \& U2 over cold plate; Use Gap Pad
- Better input protection of U2..... $\Rightarrow$ Add diode protection of inputs
- Medium-Impact Issues
- Address stability.................... $\Rightarrow$ Feedback completely redesigned
- Improve trip circuitry
$\Rightarrow$ Simplify secondary; OVP \& OCP Logical OR
- Power sequencing..... $\Rightarrow$ New regulator with programmable delay
- No kludges
$\Rightarrow$ No kludges in production
- Fabrication and soldering quality $\Rightarrow$ Will use approved vendors
- Tantalum capacitors............... $\Rightarrow$ Use 25V caps on 15V sec. out., not $16 \mathrm{~V}$
- Non-Critical But Highly Desirable
- Start-up Pulse current.
$\Rightarrow$ New regulator with programmable delay
- Eliminate pre-loads
$\Rightarrow$ No preloads $\rightarrow$ New feedback design
- Improve monitoring circuitry..... $\Rightarrow$ Differential techniques
- IPC specs for assembly
$\Rightarrow$ Design adheres to IPC specs
- Reduce/improve tuning...
$\Rightarrow$ Tuning of OVP now 1 step process

Improved

Performance

Switching Power Supply for ATLAS TileCal - G. Drake - TWEPP - Oxford, England - Sept. 20, 2012 


\section{Radiation Tests of Bricks}

- Needed to redo radiation tolerance studies

$\Rightarrow$ We used same parts, but was something missed?

$\Rightarrow$ Requested by Atlas review committee...

- Requirements:

\begin{tabular}{|c|c|c|}
\hline Photons (TID) & Neutrons (NIEL) & Protons (SEU \\
\hline $37 \mathrm{KRad}$ & $6 \mathrm{E} 12 \mathrm{n} / \mathrm{cm} 2-\mathrm{sec}$ & $1.7 \mathrm{E} 12 \mathrm{p} / \mathrm{cm} 2-\mathrm{sec}$ \\
\hline Safety Factor: 70 & Safety Factor: 20 & Safety Factor: 20 \\
\hline
\end{tabular}

- We performed 8 Sessions - Dec. 2010 - Apr. 2012

- Photons (TID) @ BNL, 1.2 MeV from Co-60

- Neutrons (NIEL) @ UMass-Lowell, U-235 reactor

- Protons (SEE) @ Mass Gen, 200 MeV radiation treatment

- Results:

- Photons: Slow degradation; No failures

- Neutrons: Slow degradation, No failures

- Protons: Found Single Event Upset with controller chip

- Further study of SEU

- Energy scan found dependence on energy (SEUs not seen before in tests at $60 \mathrm{MeV}$ )

$\Rightarrow$ We found a fix to the SEU problem - Confirmed

$\Rightarrow$ SEU energy dependence not part of specs!!!

$\Rightarrow$ Have now developed tools \& techniques to make these measurements in the future

Switching Power Supply for ATLAS TileCal - G. Drake - TWEPP - Oxford, I

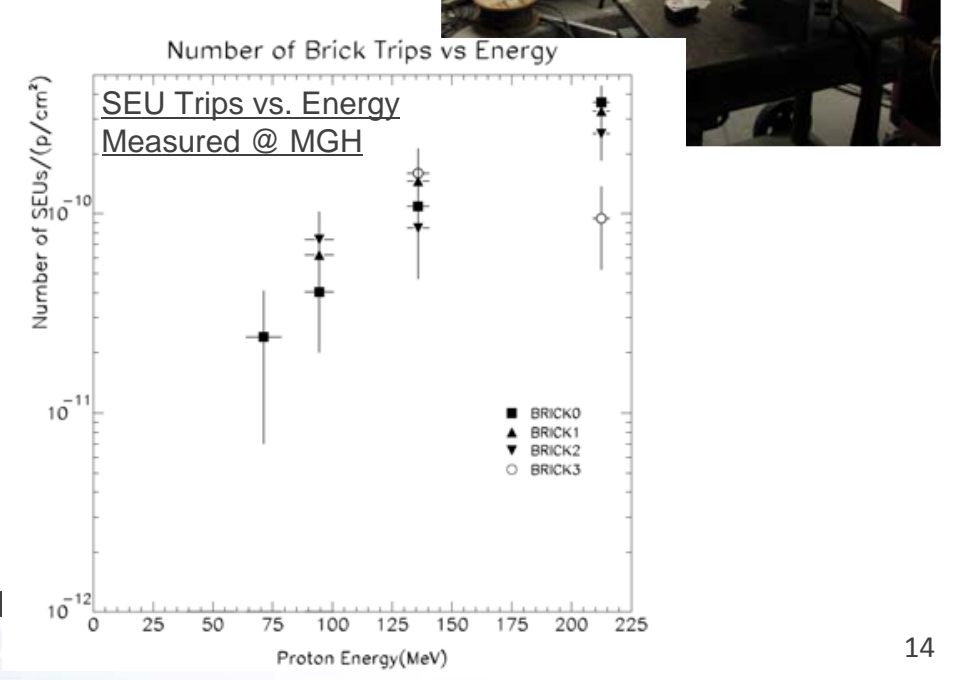




\section{The SEU Problem}

- What is happening:

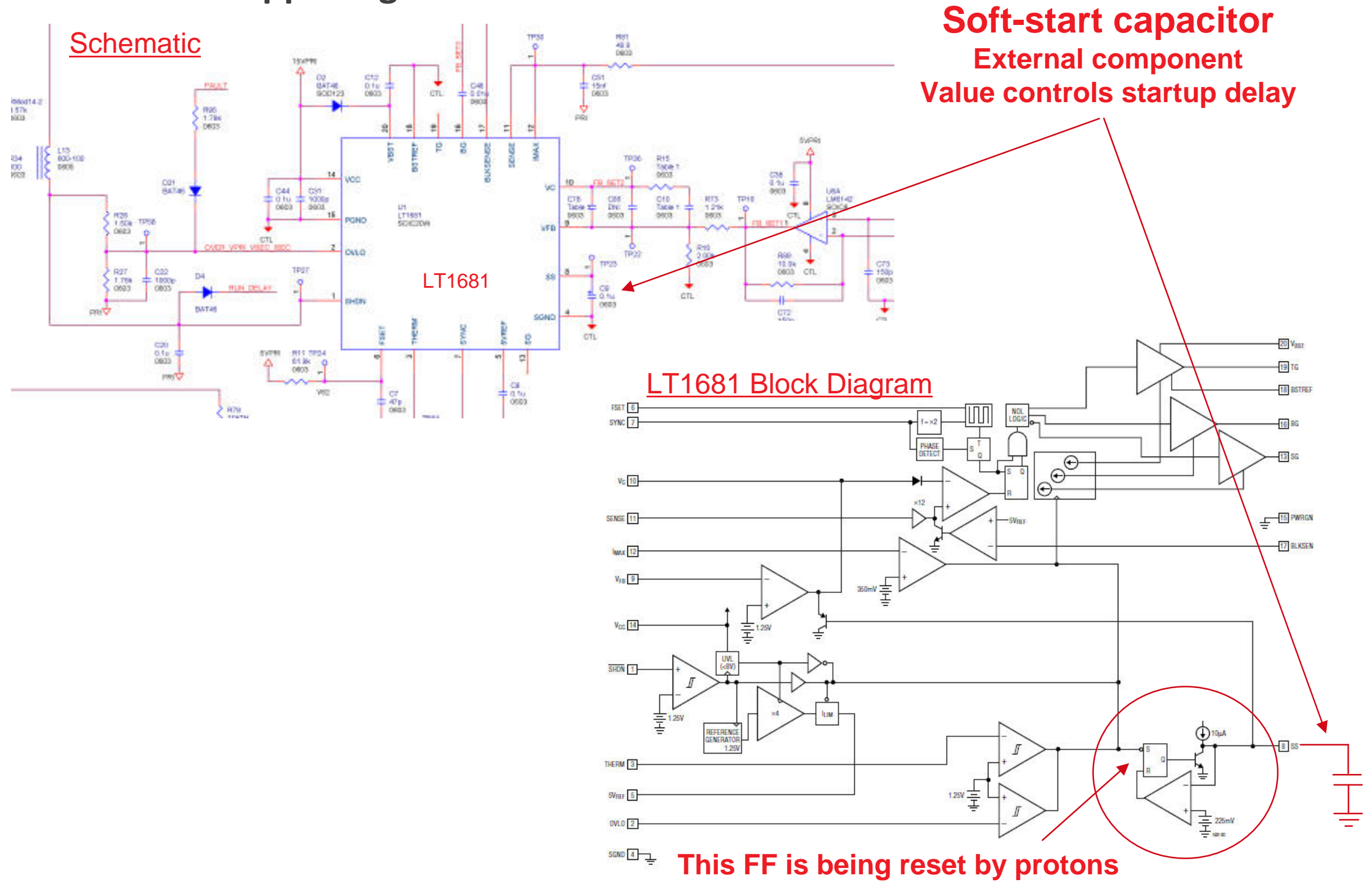

Switching Power Supply for ATLAS TileCal - G. Drake - TWEPP - Oxford, England - Sept. 20, 2012 


\section{The SEU Problem (Cont.)}

- Solution to the SEU Problem

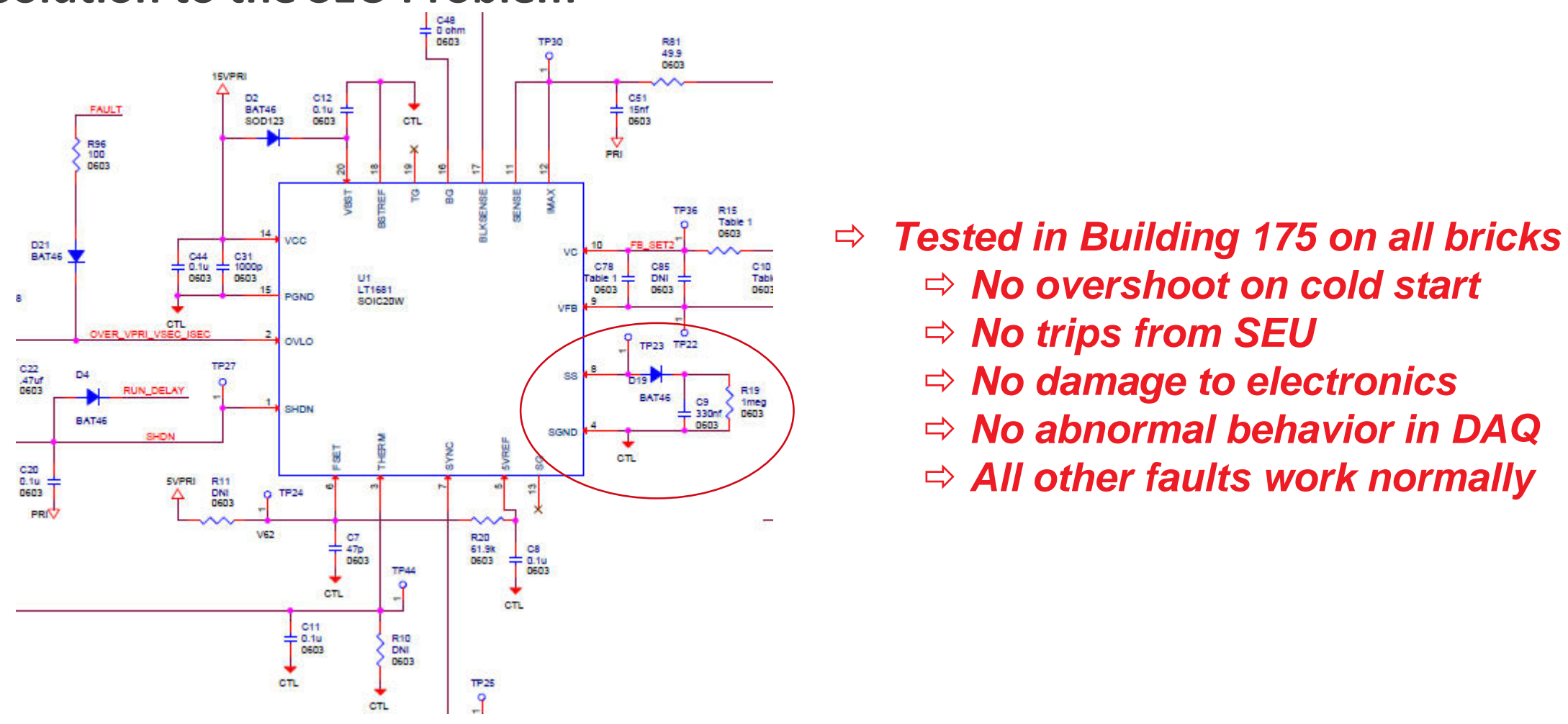

- An elegant solution to a nasty problem, requiring only 2 additional parts

- Diode is forward biased during cold start $\rightarrow$ Normal startup

- Diode is reverse biased for an SEU $\rightarrow$ Fast recovery since Cap 0

$\Rightarrow$ Has been incorporated into final design, V7.5.0 


\section{Final SEU Testing at MGH}

- Session: April 1, 2012

- Energy scan: $60 \mathrm{MeV}, 80 \mathrm{MeV}, 100.5 \mathrm{MeV}, 140 \mathrm{MeV}$, and $216 \mathrm{MeV}$

- Used (4) V7.5.0 bricks without SEU fix

- Preliminary Results:

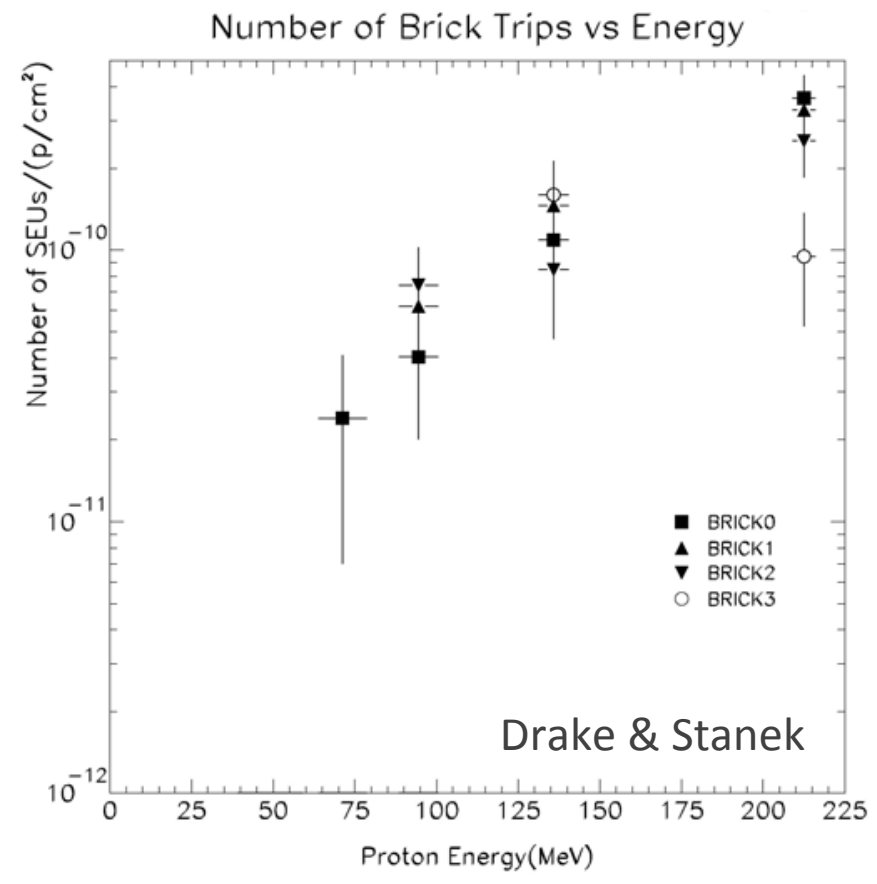

$\Rightarrow$ Can clearly see an energy threshold...

$\Rightarrow$ Analysis continuing... $\rightarrow$ Collaboration with UW-Madison

$\Rightarrow$ Will publish an Atlas Note shortly 


\section{Final Photon Testing at BNL}

- Session: April 3, 2012

- The test was run over a period of 13 hours

- Accumulated a total dose of 37 krads

- The output voltages and currents were monitored continuously through this period

- Modest changes to some of the monitored values were observed

- The inflection point in the plots seen at about $37 \mathrm{krad}$ corresponds to the time the source was closed

- There were no hard failures during the test
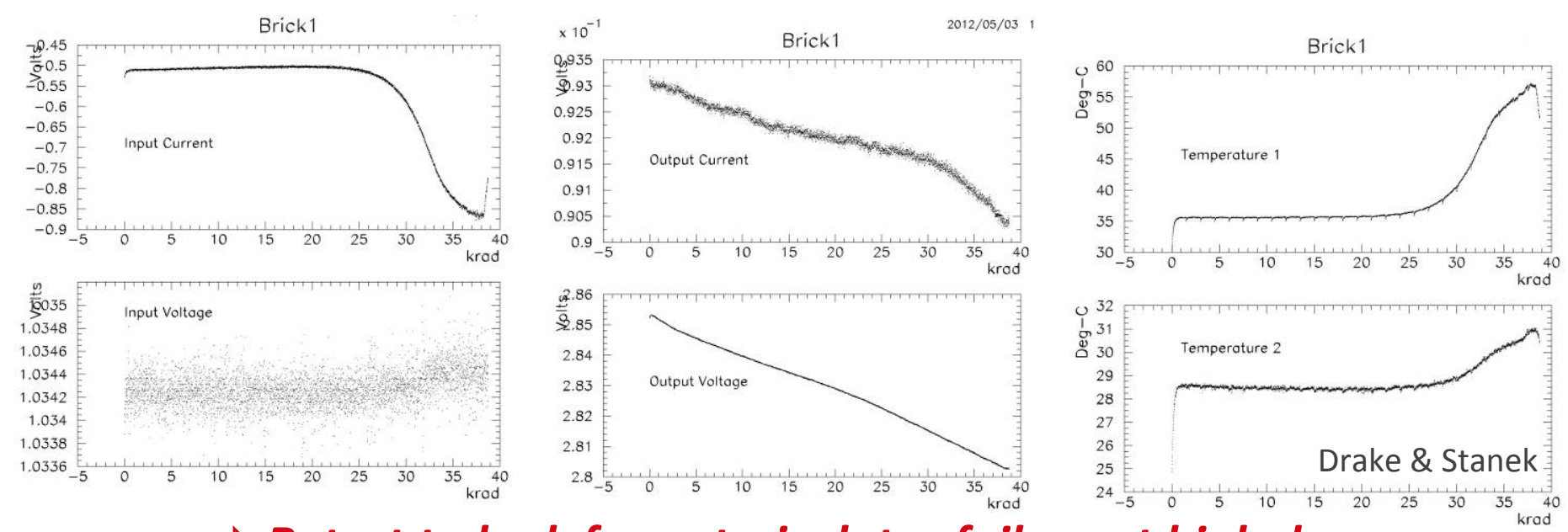

$\Rightarrow$ Retest to look for opto-isolator failure at high dose

$\Rightarrow$ Found none $\rightarrow$ OK

$\Rightarrow$ Atlas Note forthcoming

Switching Power Supply for ATLAS TileCal - G. Drake - TWEPP - Oxford, England - Sept. 20, 2012 


\section{Further Studies}

\section{- SEU Energy Dependence}

- Theory: Bendel Parameters

$\Rightarrow$ Well known in space instrumentation

$\Rightarrow$ Not well known in HEP instrumentation

- Formed research project: ANL \& UM-Madison + students

- ANL: J. Proudfoot, G. Drake, R. Stanek

- UW: Bruce Mellado, Abhirami Senthilkumaran, Anusha Gopalakrishnan

- Study \& characterization of SEU effect in semiconductors underway

- Function of feature size of devices

- Expect that this will influence future radiation tolerance specs

$\Rightarrow$ Paper coming at 2012 IEEE NSS

- Reliability

- The power supplies have had a history of reliability problems

- Single point failures, no redundancy

- No analytical study done on reliability of circuitry

$\Rightarrow$ Typically not done in HEP instrumentation either

- New bricks expected to be better but no analysis done

- Formed research project: ANL \& UM-Madison + students

- ANL: J. Proudfoot, G. Drake, R. Stanek

- UW: Bruce Mellado, Abhirami Senthilkumaran, Anusha Gopalakrishnan

- Study \& characterization of failure rates, MTBF

- Expect that this will influence future design methodology

$\Rightarrow$ Paper coming at 2012 IEEE NSS

\section{Device Cross-section $(\sigma)$}

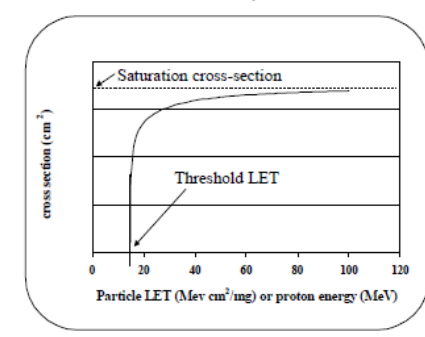

Proton beams:

$\sigma=\frac{N_{\text {events }}}{\Phi}\left(\mathrm{cm}^{2}\right)$

Heavy ion beams:

$\sigma=\frac{N_{\text {events }}}{\Phi \cos \theta}\left(\mathrm{cm}^{2}\right)$

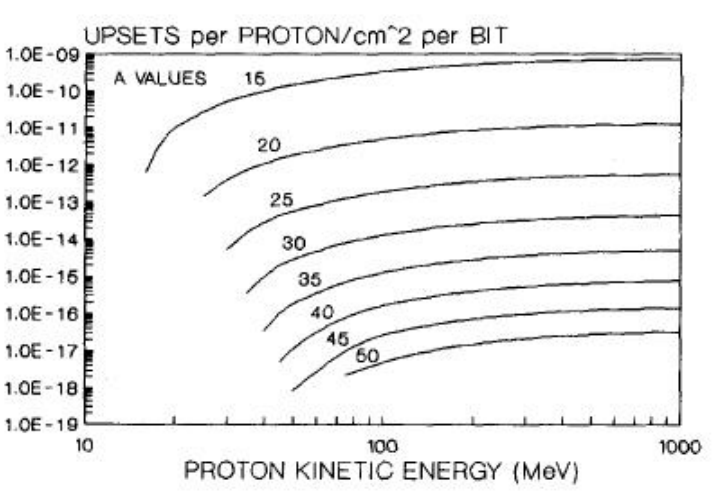

Stapor et al., IEEE-TNS, Vol 37-6, 1990

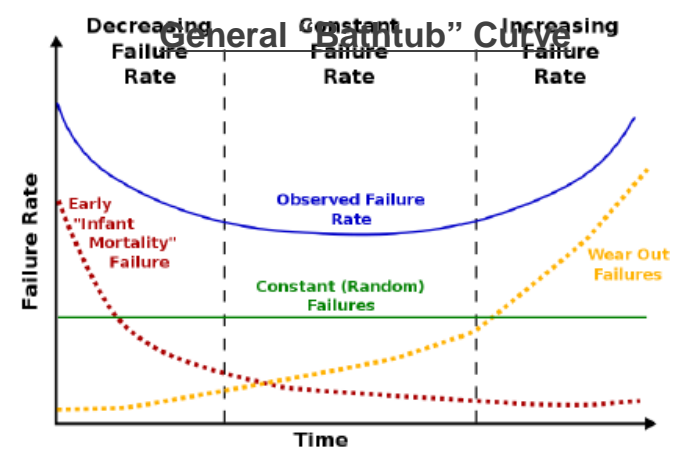

Our result: Probability of Failure-free operation:

\begin{tabular}{|c|c|c|c|}
\hline$R(t=2$ years $)$ & $R(t=5$ years $)$ & $R(t=10$ years $)$ & $R(t=20$ years $)$ \\
\hline 0.993 & 0.982 & 0.965 & 0.932 \\
\hline
\end{tabular}

$\Rightarrow$ Expect $\sim 2$ failures per year

Switching Power Supply for ATLAS TileCal - G. Drake - TWEPP - Oxford, England - Sept. 20, 2012 


\section{Project Summary to Date}

- Project Time Line

- Prototype design cycle begun June, 2009

- V7.1.0 - December, 2009, Quantity 10 bricks

- Tested at CERN in Box 066

$\Rightarrow$ Ran in long-term test since Mar. $2010 \rightarrow \sim 1.5$ yrs

$\Rightarrow$ No significant problems, Now stopped Box $0_{p}^{*}$

- V7.3.1 - November, 2010, Quantity $64(\rightarrow 80)$ bricks

- Delivered 5 boxes to CERN ( 1 from Oct. $=5$ boxes total)

- Tested at CERN, "medium-term" test, 1-2 months

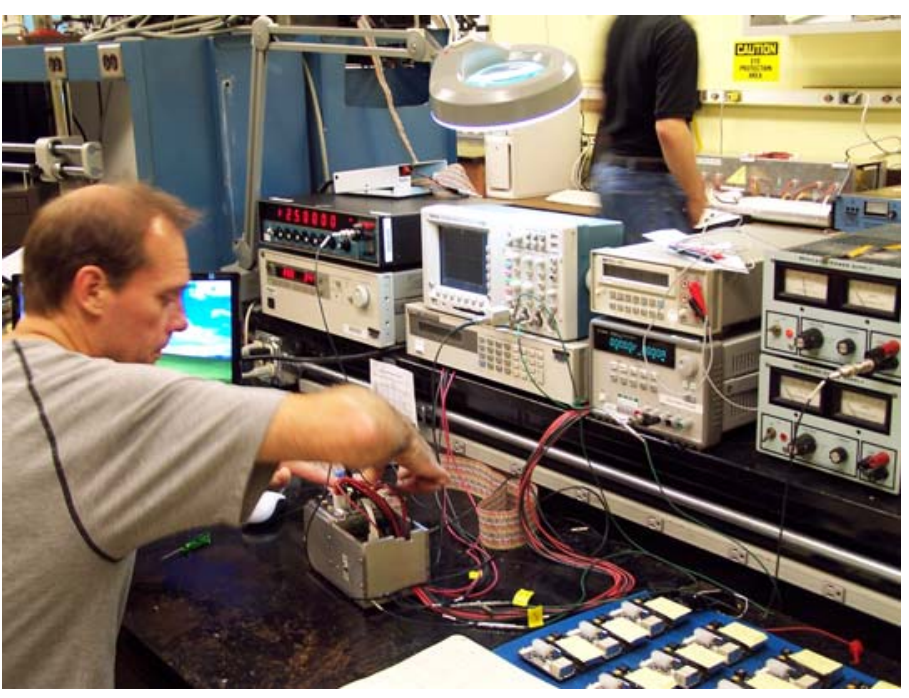

- Boxes 1-5 installed on detector during Dec. 2010 shutdown

$\Rightarrow$ Ran in long-term test Nov.-Dec., $2010 \rightarrow \sim 30-45$ days

$\Rightarrow 4$ trips on detector to date $\rightarrow \sim 600$ days (Real SEU?...)

- Production Readiness Review - Aug. 17, 2011

- V7.5.0 - December, 2011, Quantity 320 bricks

- Delivered 40 boxes to CERN

- Installed on detector

Boxes 6-45

- $\rightarrow$ Total of 45 boxes on detector

$\Rightarrow 1$ trip on detector to date $\rightarrow \sim 240$ days

Boxes $1_{p}-5_{p}$

V7.5.1 - January, 2012-present, Quantity 2080 bricks

- We are in production now, nearly complete

$\Rightarrow$ Will install remaining supplies during 2013 shutdown

Switching Power Supply for ATLAS TileCal - G. Drake - TWEPP - Oxford, England - Sept. 20, 2012 


\section{Performance on Detector}

- Trips in detector by module

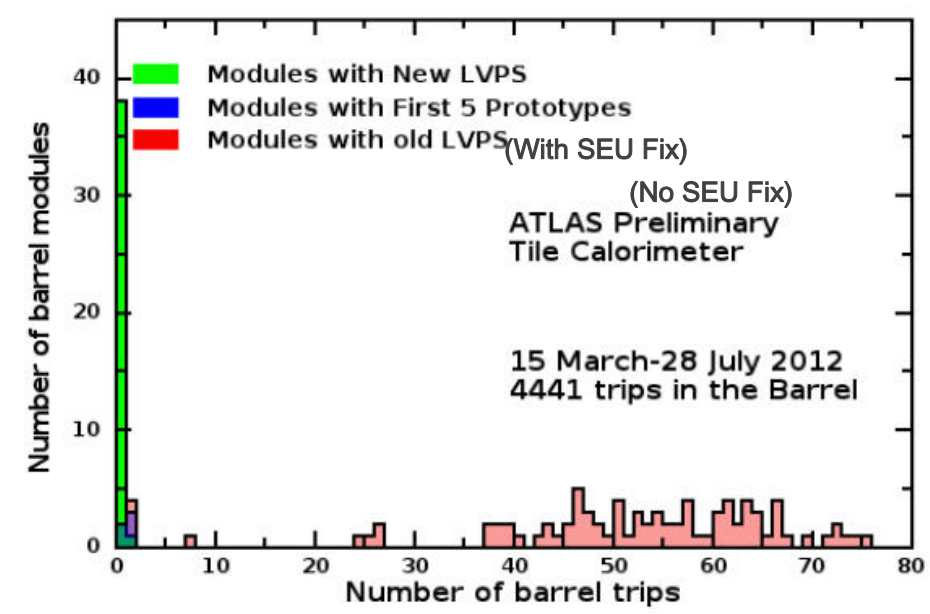

$\Rightarrow$ Current Numbers (as of Sept. 1, 2012):

$\Rightarrow 8404$ trips of old bricks

$\Rightarrow 4$ trips of pre-production boxes (5 on detector)

$\Rightarrow 1$ trip of production boxes (40 on detector)

- Noise Comparison - RMS/ $\sigma$

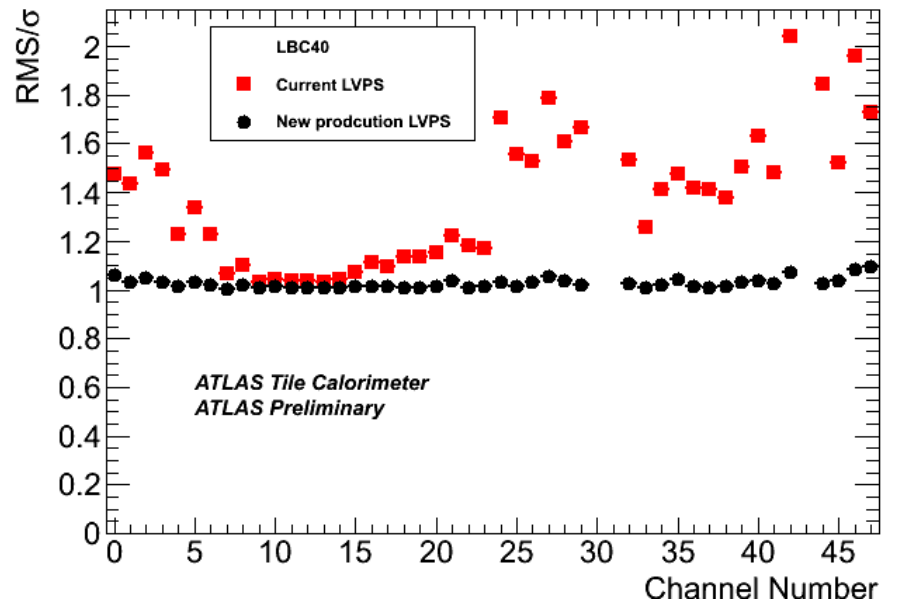

EBC39 high gain correlation 2011-03-05

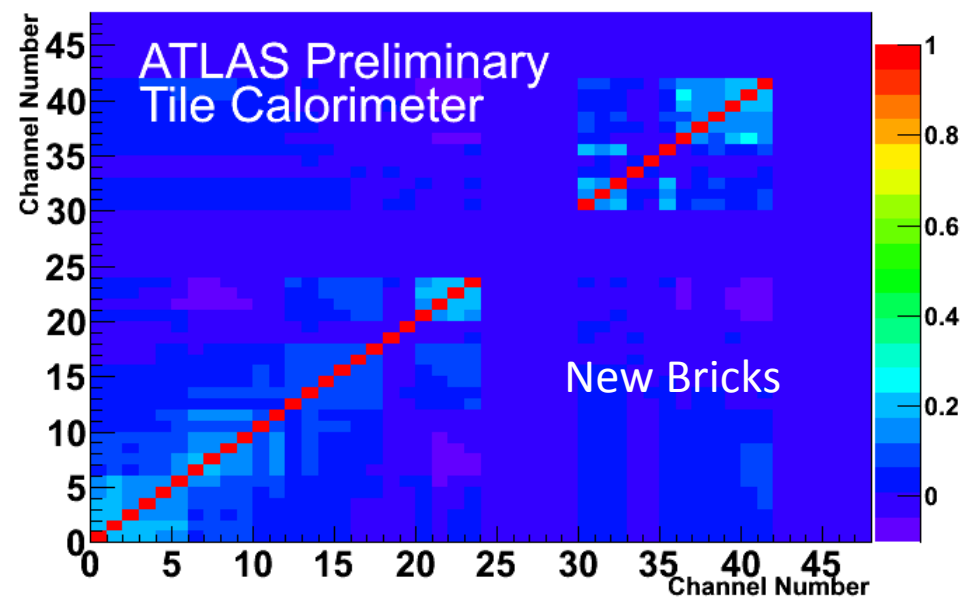

Switching Power Supply for ATLAS TileCal - G. Drake - TWEPP - Oxford, England - Sept. 20, 2012 


\section{Production Testing of Prototypes at Argonne}

- Test List:

- Frequency response $\&$ stability

- Voltage range (@ minimal load)

- Voltage range (@ nominal load)

- Stable operating range vs. voltage

- Stable operating range vs. current

- Output voltage vs. output current

- Voltage trip level vs. load

- Current trip level vs. load

- Output noise vs. output voltage

- Output noise vs. output current

- Output noise frequency spectrum

- Clock duty factor vs. output voltage

- Clock jitter vs. output voltage

- Clock duty factor vs. output current

- Clock jitter vs. output current
- Voltage monitor output vs. output voltage

- Current monitor output vs. output current

- Input voltage monitor read-back

- Input current monitor read-back

- Temperature monitor read-back

- Burn-in @ nominal load

- Check for abnormal tripping

$\Rightarrow$ Multiply by X8... Tests needed for each brick type

$\Rightarrow$ Goal for production: -0.5 hrs./brick (Not all tests, not as extensive...)

$\Rightarrow$ Box assembly \& QA additional time 


\section{Brick Checkout}

- Block Diagram of New Test Stand

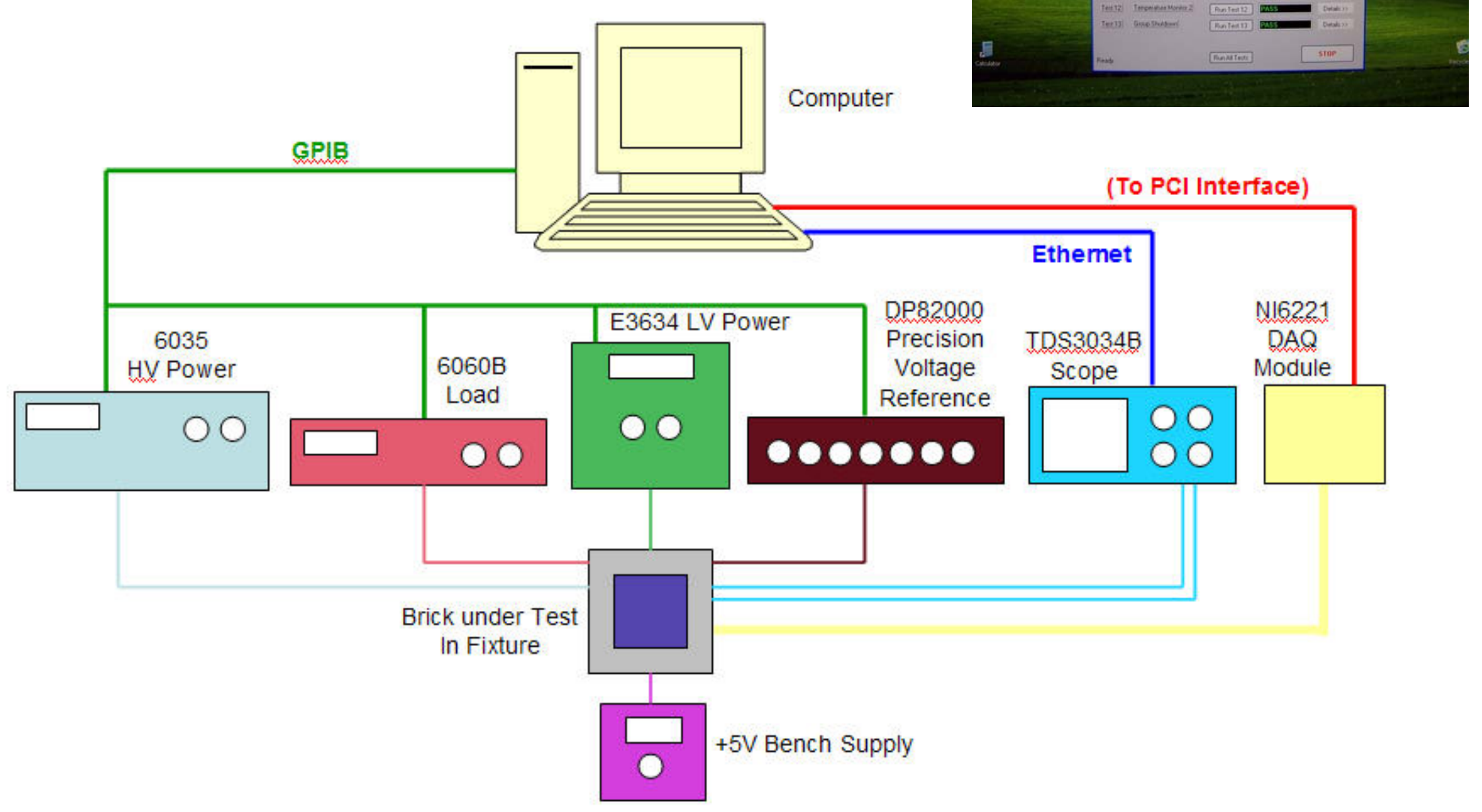

Switching Power Supply for ATLAS TileCal - G. Drake - TWEPP - Oxford, England - Sept. 20, 2012 


\section{Schedule \& Manpower}

\section{- Main production schedule - $\mathbf{2 6 0}$ boxes}

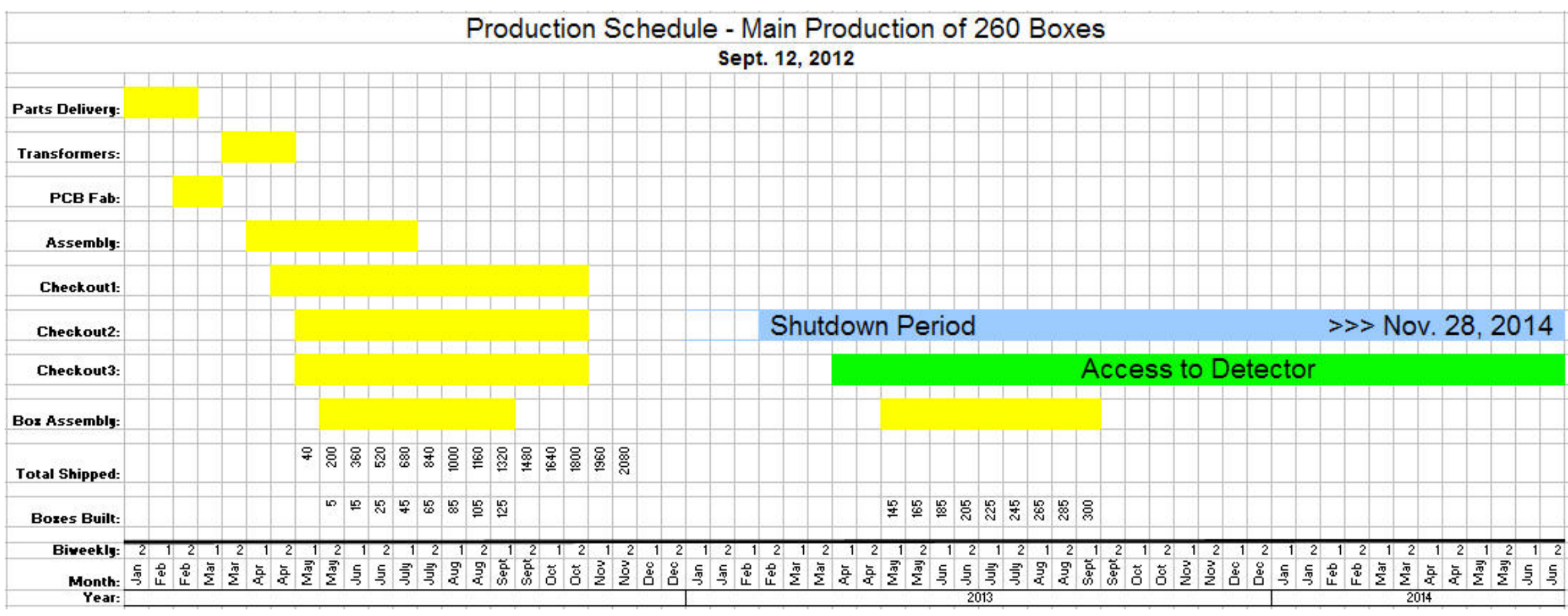

Switching Power Supply for ATLAS TileCal - G. Drake - TWEPP - Oxford, England - Sept. 20, 2012 


\section{Summary}

- Redesign project of ATLAS TileCal front-end power supplies is nearing completion

- Begun in 2009

- Steady progression of prototype development, pre-production, and production

- Will have full replacement complete in 2013 shutdown

- New design addresses several performance issues

- Noise

- Latch-up of opto-isolators

- Thermal management

- SEUs in controller chip

- Tripping as a function of luminosity

- + others...

- Current status of $\mathbf{4 5}$ boxes on detector

- Only 4 trips of pre-production boxes in $\sim 600$ days

$\Rightarrow$ Probably real SEUs...

- Only 1 trip of production boxes in 240 days

- Radiation Tolerance Measurements \& Analysis

- Tolerance to photons \& neutrons OK

- Discovered SEU problem with controller chip

- SEU probability has energy dependence, $\sim 80 \mathrm{MeV}$ threshold

$\Rightarrow$ Not specified in radiation tolerance specs...

- Problem has been addressed with circuit modification

- Study under way to incorporate theoretical Bendl Curves

- Reliability analysis

- Have developed reliability models for the new power supply

- Expect 2 failures per year

- Next Gen: Redundancy

- Production checkout is nearing completion at Argonne

- 2400 bricks total, 300 boxes

- 45 boxes installed on detector to date; Performance looks very good

- On track to complete on time \& on budget

$\Rightarrow$ LVPS Replacement should significantly improve the performance of the TileCAL Detector

Switching Power Supply for ATLAS TileCal - G. Drake - TWEPP - Oxford, England - Sept. 20, 2012 